\title{
CHARACTERIZATION OF EFFLUENTS GIVEN OFF BY WIRING INSULATION
}

William T. Yost

K. Elliott Cramer

Daniel F. Perey

Nasa-Langley Research Center

Mail Stop 231

Hampton VA 23681-2199

\begin{abstract}
When an insulated wire is heated, the insulation emits a variety of effluents. This paper discusses the basis of emissions of effluents from wiring insulation. Several species are emitted at relatively low temperatures, while others are emitted when the wire reaches higher temperatures. We isolate the emissions by relative molecular weight of the effluents and measure the effluent concentration both as a function of time (temperature held constant) and by wire temperature. We find that the Law of Mass Action describes and predicts the time-dependence of the emission of a specific effluent caused by the heating. The binding energy is determined by performing an Arrhenius Plot on the temperature data. These dependencies are discussed and working equations are derived. Data collected from 20 gauge wire (MIL$\mathrm{W}-22759 / 11-20)$ is used to illustrate and confirm the validity of the theory.
\end{abstract}

\section{INTRODUCTION}

A simple and practical form of wire consists of a central conducting region (generally made of a conducting material such as copper) and an outer sheath (insulation) generally made of a stable non-conducting material. One usually cuts the wire to length, installs it, and dresses and bundles it with other wires so that it follows the desired path. Then at each end a connector is installed to complete the cable

The insulating outer sheath plays several critical functions in preserving wiring integrity. It permits electrical isolation of conductors as well as adding some measure of mechanical stability. However it can suffer damage from numerous causes. Hence it becomes the first line of defense to assure appropriate wiring function.

Depending on the application and care followed in the installation the wire is generally regarded as a trouble-free component. However, when exposed to severe environmental conditions, such as high temperatures, high ultraviolet light flux, various sprays, greases, and hydraulic fluids, the insulation deteriorates. Improper or careless cable placement can result in chafing, ultraviolet-induced damage, and or thermal damage. 
Electrically induced damage can also affect wiring integrity. Surges (both over-voltages and over-currents) and regional electrical discharge can cause the insulation to be compromised. In some cases the wiring insulation becomes brittle with age. Any flexing then results in the physical break-up and detachment of the insulator from the conductor. With the central conductors exposed, short circuits can lead to functional failure. In some cases the failure can be catastrophic resulting in loss of mission objectives, mission failure or even loss of life.

Functional tests coupled with visual inspection are presently used to verify that wiring is in good working order. However, a thorough visual inspection requires that wiring bundles be separated and detached from harness fixtures. This requires physical manipulation, which can cause further damage to the insulation and result in cracking and flaking.

In this paper we explore part of the science base of a promising approach, which can determine wiring exposure to aging conditions including over-currents, arcing, and high temperatures. It is based on the fact that insulation surfaces exposed to harsh or aging environments results in emission of effluents that become gas-borne whenever severe conditions are encountered. The technique also holds the promise that an effective wire history can be obtained with the application of a short thermal cycle to wiring under investigation.

\section{THEORY}

\section{$\underline{\text { Law of Mass Action }}$}

Consider an insulator surface onto which is applied a chemical species of a specific molecular weight. Furthermore assume that this species is held to the surface by means of an attractive force. The attachment can be expressed as a chemical equation as

$$
I S \Leftrightarrow I+S
$$

where $\mathrm{I}$ is the insulator, and $\mathrm{S}$ is the species Under these conditions and for a given temperature, thermal agitation causes the release of species $S$ at a rate that is determined by its concentration. Thus we assume that at each current level, the wire experiences Jouleheating and by transfer the insulation experiences a temperature increase. We assume that at a constant current, the insulation temperature reaches an equilibrium and hence characteristic value, and therefore we apply the law of mass action[1]. The rate of species escape from the insulation surface is proportional to the concentration $\mathrm{C}_{\mathrm{s}}$, with the constant of proportionality, $\mathrm{K}_{\mathrm{s}}$ as shown in the equation

$$
\frac{d C_{s}}{d t}=-K_{s} C_{s}
$$

The solution of this gives

$$
C_{s}=C_{s i} e^{-K_{s} t}
$$

where $\mathrm{t}$ is time, $\mathrm{C}_{\mathrm{si}}$ is initial emitted concentration. In this treatment we have assumed that once the dissociation occurs, the effluent escapes from the insulation essentially unimpeded and is collected by a sensing device. We believe that this assumption is adequate for these data since the time of data collection is sufficiently long. 


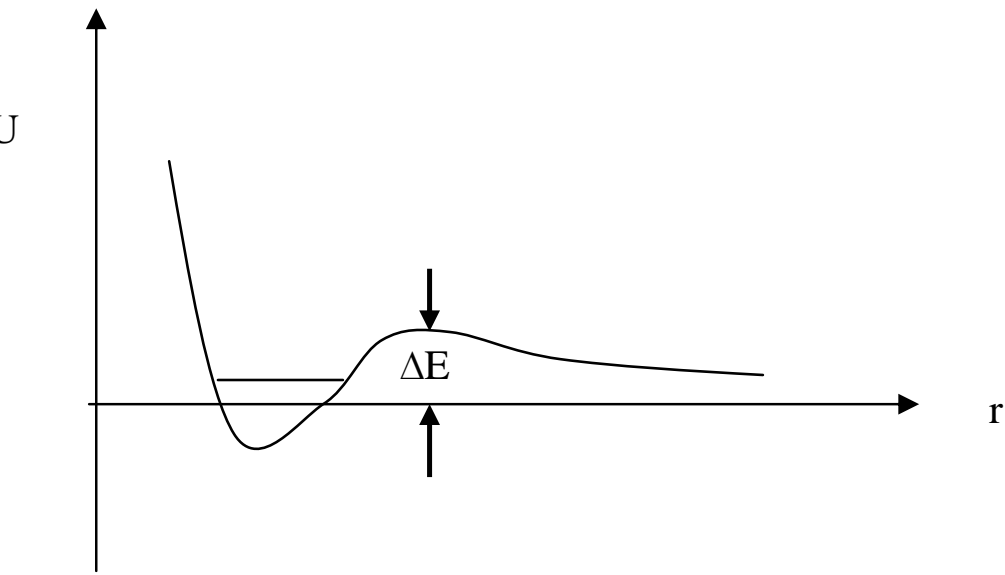

FIGURE 1.A plot of potential energy as a function of distance $r$ from the insulator surface. The attachment energy, $\Delta \mathrm{E}$, is shown as the energy that must be provided for the species to escape.

An important parameter associated with Eq. (3) is a time constant of

$$
\tau=\frac{1}{K_{s}}
$$

where $\tau$ is the time necessary for the concentration of the species to decrease to $1 / \mathrm{e}$ of its original value. This quantity is a significant factor in choosing which species is chosen to monitor the service life.

\section{$\underline{\text { Arrhenius Rate Equation }}$}

Consider the attachment energy of a molecular species as a function of distance from the insulator. Figure 1 is a schematic representation of the species energy that is attached. We represent its potential energy, $U$, as a function of distance from the insulator. Species become effluents when given off during the wire heating. They are dissociated from the insulator's molecular structure when released. If there are multiple species, then each has a different dissociation energy, and each will come off at different rates, depending on the insulator temperature. We designate the dissociation energy of each species as, $\Delta \mathrm{E}_{\mathrm{s}}$. Once the effluent has escaped from the insulator, then we further assume that it is collected and its concentration is measured.

If the concentration of the species under measurement is $\mathrm{C}_{\mathrm{osi}}$, and the concentration escaping the insulator is $\mathrm{C}_{\mathrm{si}}$, then we write the Arrhenius Equation [2]

$$
C_{s i}=C_{o s i} e^{-\frac{\Delta E_{i}}{k T}}
$$

where $\mathrm{T}$ is the temperature in kelvins and $\mathrm{k}$ is Boltzman's constant.

If we assume that at each current level, the wire and insulation temperature reaches an equilibrium value, then we rewrite Eq. 5 as 


$$
\Sigma C_{s i}=\Sigma\left(C_{o s i} e^{-\frac{\Delta E_{i}}{k T}}\right)
$$

where the sum is over all species. This gives the total effluent flux as a function of temperature, where emission of multiple species is involved.

\section{EXPERIMENTAL CONFIRMATION PROCEDURE}

Experimental details are covered more extensively in [3]. We give a brief synopsis here for completeness. We chose 20 gauge copper stranded (MIL-W-22759/11-20) wire with polytetrafluroethylene (PTFE) insulation for these tests because of its wide use. When used in open air, 20 gauge (AWG 20) copper wire is rated to carry 11 amps, whereas in more restrictive environments, such as open wiring bundles, the recommended maximum current should not exceed 7.5 amps [4]. In applications to chassis wiring the maximum current generally falls between 2 and 3 amps. The current rating decreases as environmental cooling capability decreases.

Five identical wire specimens were prepared from a continuous length. A $128.3 \mathrm{~cm}$ length of wire was cut, cleaned with methanol, and inserted into a $40 \mathrm{ml}$ glass vial. The ends of the wire were passed through a septum at the end of the vial and connected to a computer controlled DC power supply.

The temperature of copper influences the wire resistance. The resistivity of copper rises by $32 \%$ as copper rises from $20 \mathrm{C}$ to $100 \mathrm{C}$. Typically, 20 gauge wire varies from 0.0324 ohms/meter at $20 \mathrm{C}$ to $0.0429 \mathrm{ohms} /$ meter at $100 \mathrm{C}$. Hence our samples vary in resistance from $0.0416 \mathrm{ohms}$ to greater than $0.0550 \mathrm{ohms}$. The heat power generated in these samples reached a maximum above 4.96 watts at 9.5 amps. At these values the wire temperature is measured in the measurement vial to be a maximum of $58 \mathrm{C}$ at 9.5 amps. For simplicity we assume a uniform temperature for the wire.

Effluent measurement was taken with a portable fast gas chromatography system [5]. The $\mathrm{zNose}^{\mathrm{TM}}$ is an electronic system that uses an uncoated $500 \mathrm{MHz}$ surface acoustic wave (SAW), detector to determine molecular concentration. Input vapors enter the system through a temperature-controlled inlet and are pre-concentrated for a specific period of time. These vapors are then injected as a short pulse into a temperature controlled capillary column for separation by molecular weight. The column of separated effluents is then deposited onto the SAW detector, which records the time and amount of each effluent as a shift in the resonant frequency. In this way one obtains a relative concentration of each species that is given off by the wire.

\section{RESULTS}

Figure 2 shows the result from measuring the effluent flux as a function of time while holding temperature constant. Error bars on the data points represent the standard deviation of a series of measurements on different samples. From the graph we show agreement with Equation (3) with $\mathrm{K}_{\mathrm{s}}=6.8 \times 10^{-4} / \mathrm{min}$. The agreement with the theory $\left(\mathrm{R}^{2}=0.97\right)$ is good.

In some applications the instrument is unable to separate out two different effluents of similar molecular weight. In general each will have a different $\Delta \mathrm{E}$ even though they are of similar molecular weight. In this case one subtracts out the longer lived (higher $\Delta \mathrm{E}$ ) effluent from the short lived one. Figure 3 shows the result of such a subtraction. The error bars reflect the standard deviation of the data points after subtraction of the average of the longlived effluent. We point out that the subtraction isolation of the short-lived member gives good agreement with Equation (3). 


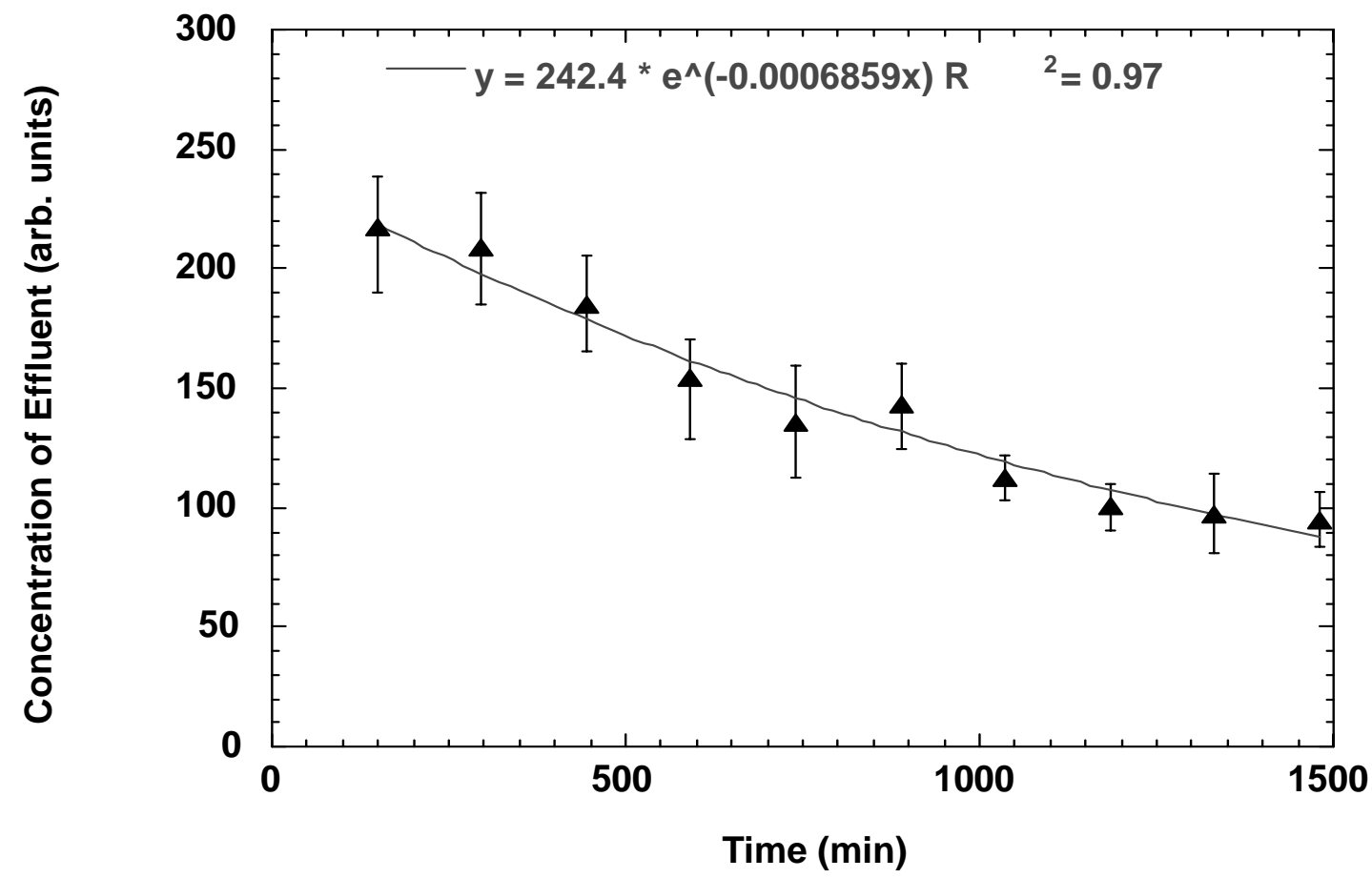

FIGURE 2. A plot of concentration of effluent as a function of time, while holding temperature of wire constant.

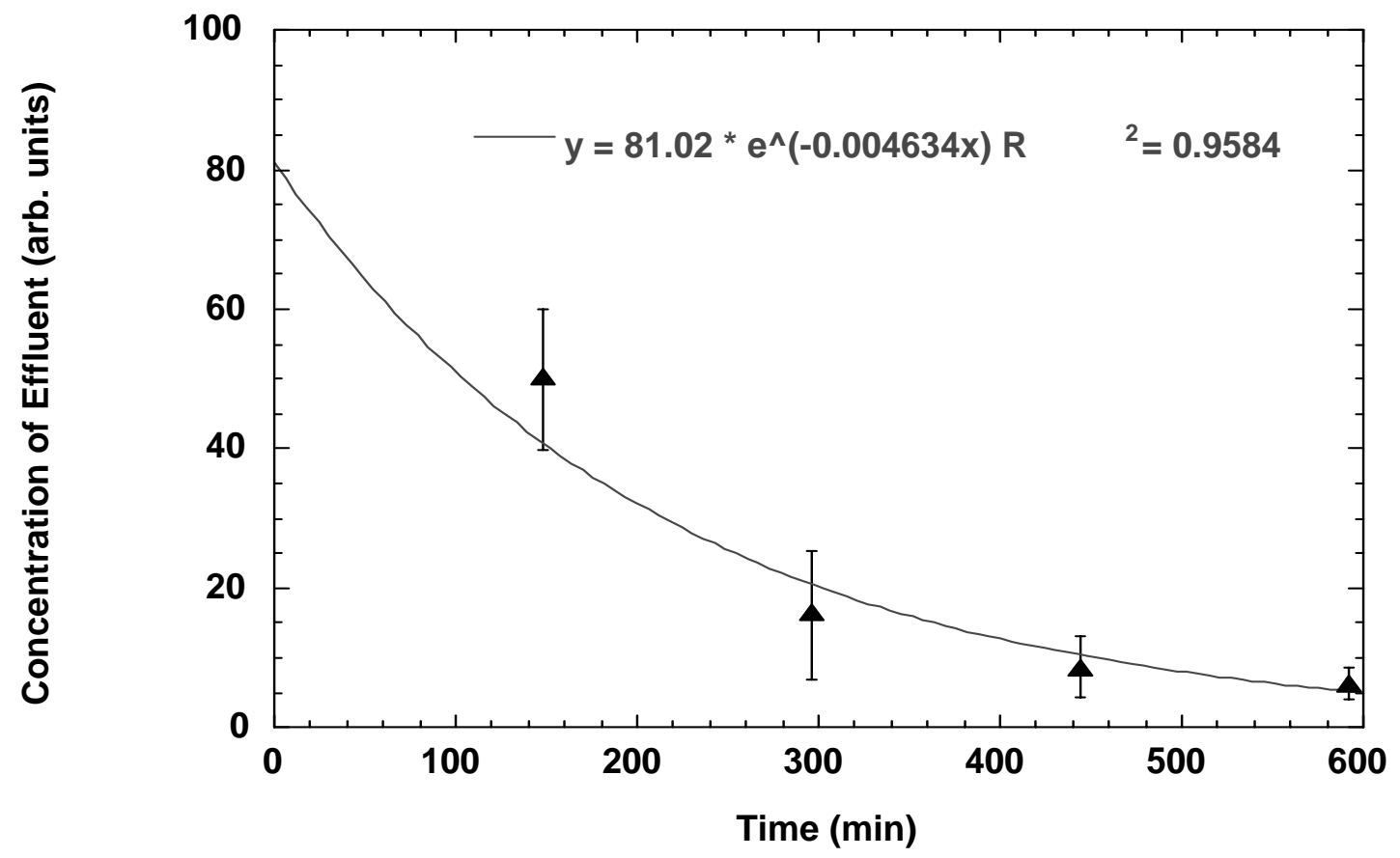

FIGURE 3. A plot of a short lived effluent obtained from two effluent concentrations of similar molecular weight while holding temperature constant. The long-lived effluent (higher $\Delta \mathrm{E}$ ) was removed by subtraction. 


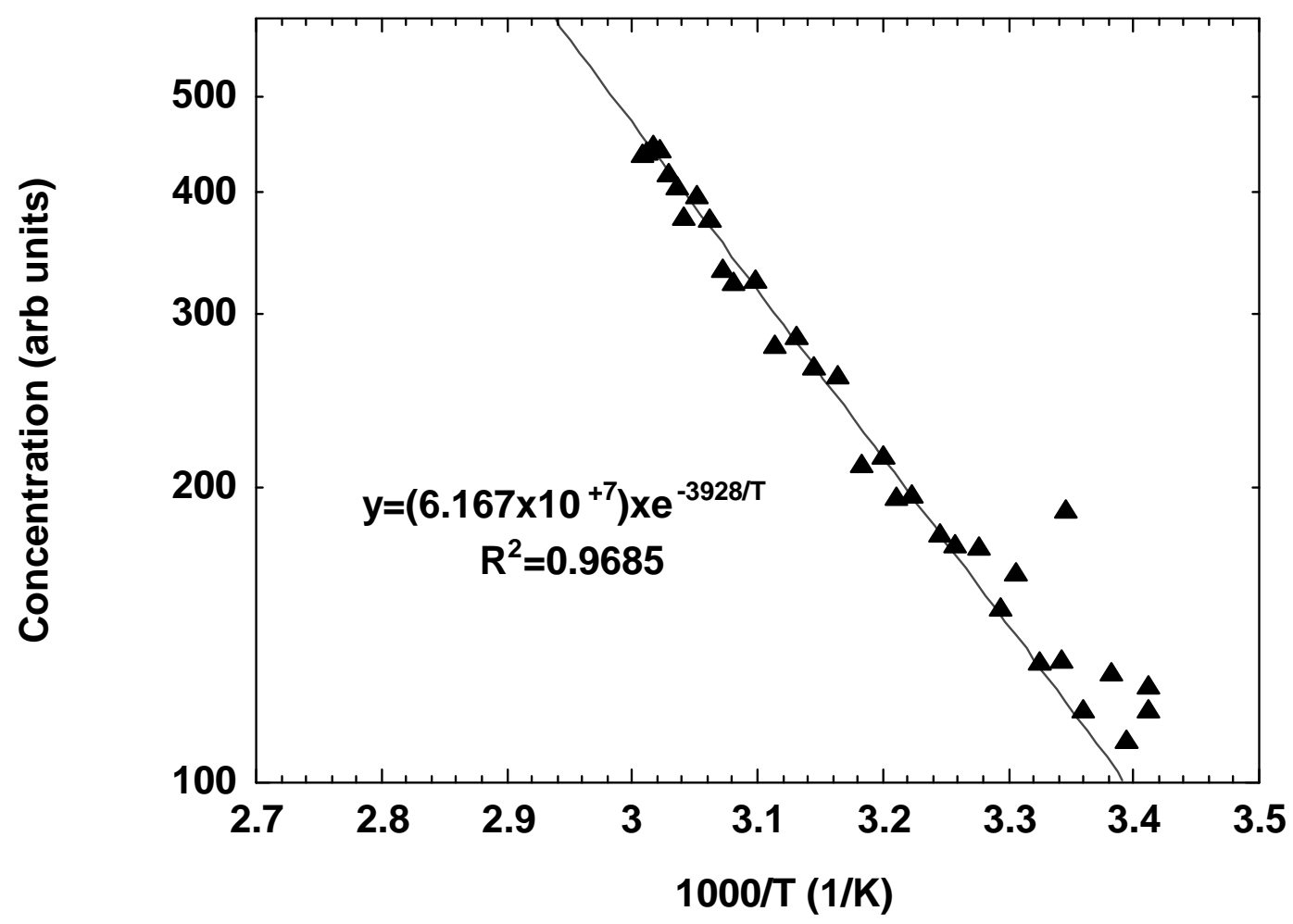

FIGURE 4. A plot of concentration vs. $1000 \log (1 / \mathrm{T})$, where $\mathrm{T}$ is temperature.

According to Equation (5), a plot of log concentration vs. (1/T) where $\mathrm{T}$ is temperature in kelvins gives the value of $\Delta \mathrm{E}$. In this case we selected a single effluent and monitored the concentration given off as the wire temperature was changed. We chose a long-lived effluent whose $\Delta \mathrm{E}$ was sufficiently long that time-related effects could be minimized from the data. When the temperature varies, one notices the exponential character of the plot as predicted by Equation (5).

Figure 4 is a typical plot of concentration of emission of effluent of a selected molecular weight as a function of reciprocal temperature. The straight line shown gives the coefficient $\alpha$ of 3928 in the exponent. Determination of this coefficient allows the calculation of $\Delta \mathrm{E}$ by the relationship

$$
\alpha=\frac{\Delta E}{k}
$$

and permits the calculation of the attachment energy $\Delta \mathrm{E}$ for this effluent to be $0.338 \mathrm{eV}$. We estimate the uncertainty to be approximately $\pm 0.09 \mathrm{ev}$.

\section{CONCLUSIONS}

We have demonstrated that it is possible to segregate and measure the concentration of effluents given off from wires when heated. From information obtained with this technique 
we have been able to determine the attachment energy to the insulator. We also have shown that it is possible to determine "aging" effects related to overheating and the like.

By using a device that can sort effluents by molecular weight and measure their concentration it is possible to determine species given off from wiring insulation. This result allows for the development of a NDE device that can monitor the state of the insulators of wiring. By adding certain species or "markers" that attach to the insulator surfaces, one can engineer NDE-friendly wiring that will allow for the continual assessment of insulator status and hence, "health" of wiring. It has the possibility of special application to wiring bundles, where each wire in the bundle will have its own unique markers. In this way one can determine which wire has experienced current overloads or other possible damaging insults simply by monitoring the effluents. Another possible use is the determination of chemical exposure by classifying any chemical that may attach to the insulator (If it does not attach, chemical attack is not likely to be a problem).

\section{REFERENCES}

1. The Law of Mass Action is covered in many references. We prefer one that has a mathematical slant, such as Murray R. Spiegel, "Applied Differential Equations", Prentice-Hall, Inc. Englewood Cliffs, NJ. ,1958, pp 93-94

2. The Arrhenius Equation is generally covered in books covering chemical kinetics and also in Material Science, such as Arthur L. Ruoff, "Materials Science", Prentice-Hall, Inc. Englewood Cliffs, NJ. ,1973, pp 444-445

3. Cramer, K. Elliott, Yost,, William T. and Perey, Daniel F.,'Effluent based characterization of aerospace wiring." in Review of Progress in QNDE, earlier paper in this volume.

4. Typical uses of wiring can be found in references such as The Radio Amateur's Handbook, eds.. Rusgrove, Jay, and Woodward, George, American Radio Relay League, 1981, pp 17-6 and 17-7.

5. Staples, E. J. and Watson, G. W. , "A Gas Chromatograph Incorporating an Innovative New Surface Acoustic Wave (SAW) Detector", Pittcon Conference, 1-5 March, 1998, New Orleans LA. Paper 1538CP 